\title{
Herlyn-Werner-Wunderlich syndrome - Common presentation of an unusual variation: A Case Report
}

\author{
${ }^{1}$ Dr.Ajit Mahale,${ }^{2}$ Dr. Nina Mahale. ${ }^{3}$ Dr. K Santosh (Resident) \\ ${ }^{I}$ Professor \& Head of department, Department of radio diagnosis KMC hospital, Manipal University, Attavara, \\ mangalore, Karnataka, India. \\ ${ }^{2} M D$ Consultant gynecologist and Associate professor, KMC hospital, Manipal University, Attavara, \\ mangalore, Karnataka, India. \\ ${ }^{3} J$ r. Resident, Department of radio diagnosis KMC hospital, Manipal University, Attavara, mangalore, \\ Karnataka, India.
}

\begin{abstract}
Herlyn-Werner-Wunderlich syndrome also known as uterus didelphys with obstructing hemivaginal septum and ipsilateral renal agenesis is a rare syndrome with only a few hundred reported cases described since 1922. Uterus Didelphys is a type of lateral fusion disorder of mullerian ducts. Mullerian duct anomalies have an incidence of 2-3\%, while obstructing hemivaginal septum and ipsilateral renal agenesis constitutes 0.16-10\% of these mullerian duct anomalies. A female case referred to our radiology department for evaluation of lower abdominal pain revealed Uterus Didelphys with Obstructing Hemivaginal Septum and Ipsilateral Renal Agenesis on imaging. Understanding the imaging findings, in conjunction with the clinical presentation, is critical for early diagnosis in attempting to prevent complications such as endometriosis or adhesions from chronic infections with subsequent infertility. Management depends on the presence or absence of an obstruction. Surgical removal of the vaginal septum is the preferred approach for relieving an obstruction.
\end{abstract}

Key words: Mullerian Ducts, MRI Pelvis, Uterus Didelphys, Hemivaginal Septum, Ipsilateral Renal Agenesis.

\section{Introduction}

Uterus Didelphys with Obstructing Hemivaginal Septum and Ipsilateral Renal Agenesis, also known as Herlyn-Werner-Wunderlich syndrome is a rare syndrome with only a few hundred reported cases described since 1922. Mullerian duct anomalies have an incidence of 2-3\%. While OHVIRA (obstructing hemivaginal septum and ipsilateral renal agenesis) constitutes $0.16-10 \%$ of these Mullerian duct anomalies.

\section{Case presentation}

A 28-year-old lady is referred to our hospital investigate for lower abdominal pain \& scanty menstruation. She reported previous episodes of similar, but less severe, pain.

Physical examination revealed only mild tenderness at palpation of the middle \& lower abdomen. Findings of laboratory tests were unremarkable. Menstruation first occurred in this patient at 14 years of age and at admission, she was on the $5^{\text {th }}$ day of her menstrual cycle. On vaginal examination bulging vagina is seen. Utrasonography and Computed tomography of the abdomen is recommended for evaluation of pain.

\section{Imaging Findings}

Trans abdominal ultrasound demonstrated widely divergent uterine corpi with a large fundal cleft, each with its own endometrial cavity two cervices (images not shown) raising the index of suspicion for bicornuate bicollis uterus/ uterus didelphys anomaly. Renal evaluation revealed an absent right kidney corroborating the likely diagnosis of uterus didelphys with ipsilateral renal agenesis.

Computed tomography of abdomen \& pelvis is done to rule out any ectopic site of hypoplastic kidney $\&$ other causes of pain. CT refotmated images showed unilateral kidney \& absent right kidney (Fig 1b) 


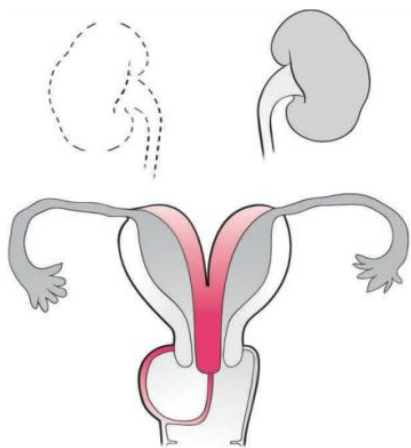

a.

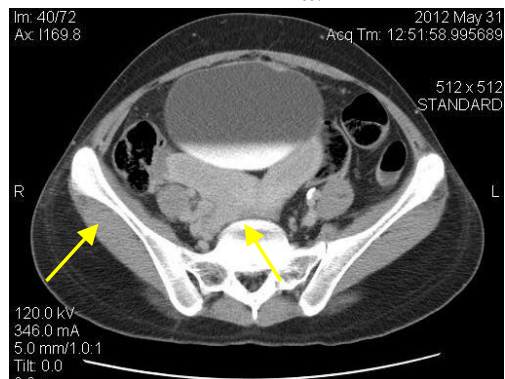

c.

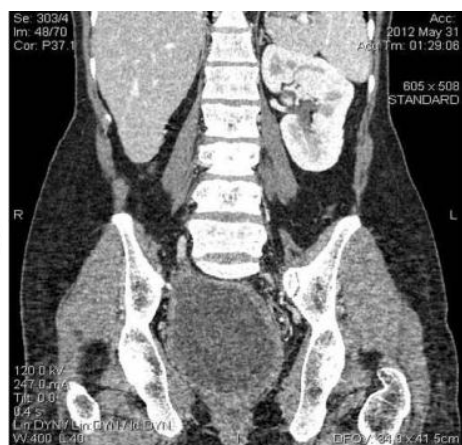

b.

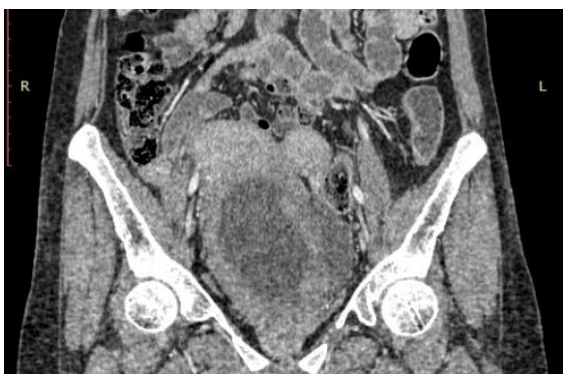

d.

FIGURE 1: (a.)graphical illustration of uterus Didelphys with Obstructing Hemi vaginal Septum and Ipsilateral Renal Agenesis. Selected CECT images of the pelvis shows - (b.) Coronal reformatted venous phase images show absent right kidney bicornuate uterus with(c,d) large fundal cleft, two uterine horns (arrows) \& cervices and thin walled longitudinal septum

To better delineate the anatomy, MRI pelvis was done. MRI images showed large fundal cleft of more than $1 \mathrm{~cm}$ consistent with uterus didelphys.

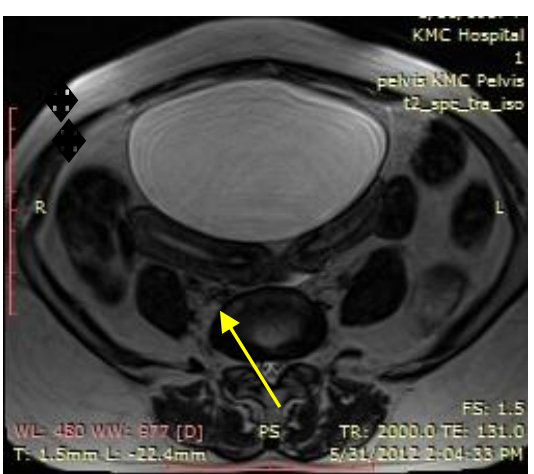

(a.)

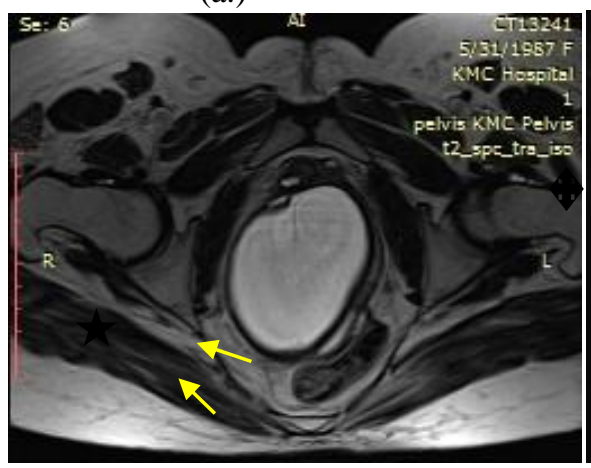

(c.)

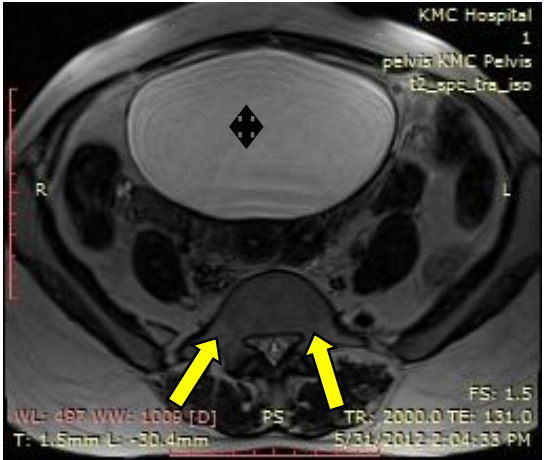

(b.)

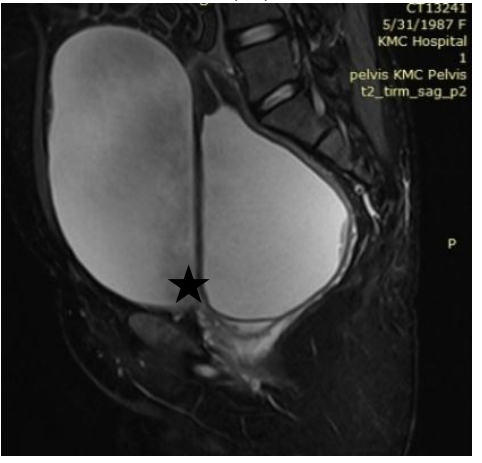

(d.) 


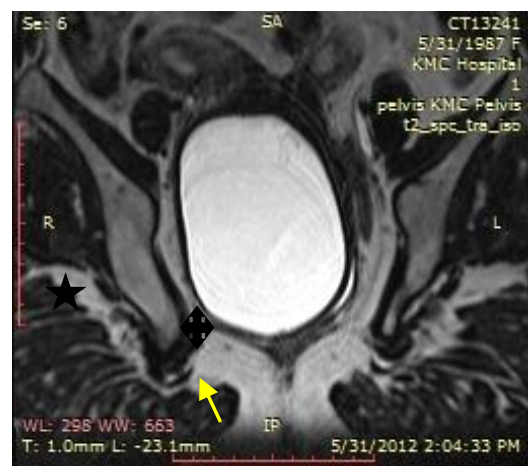

(e.)

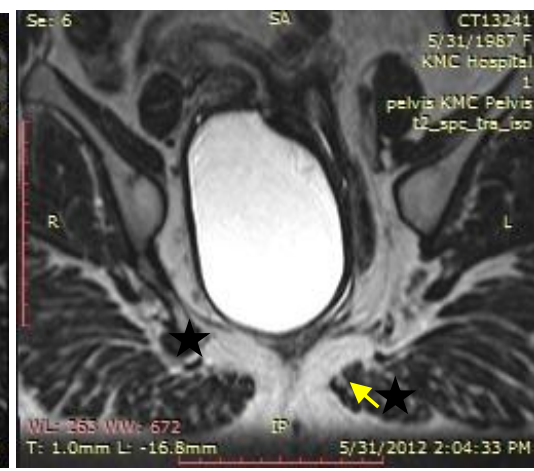

(f.)

FIGURE 2: MR T2 axial images(a,b,c) shows two uterine horns(arrows), two cervices(Block arrows) \& dilated right hemivagina with obstructing septum. MR T2 sagittal (d) and coronal images(e,f) images communication of the right uterine horn with the dilated right hemivagina( ) and compressed left vagina(small arrows). ( -

\section{Urinary bladder)}

\section{Discussion}

Uterus didelphys is a symmetric anomaly in which two completely separate uterine cavities are identified, each with normal zonal anatomy as well as its own endometrial cavity and cervix [1,2,3]. No communication between the two cavities is present. A complete or partial longitudinal vaginal septum is associated with this anomaly in $75 \%$ of cases $[4,5]$.

Some patients with uterus didelphys present with a unilateral hemivaginal septum, which may result in obstruction with consequent hematometrocolpos [4,5] The majority of cases reported in the literature are associated with renal agenesis on the same side as the obstructing transverse hemivaginal septum [4,6]. This syndrome has been referred to as obstructed hemivagina-ipsilateral renal agenesis anomaly.

Uterus Didelphys is a type of lateral fusion disorder of mullerian ducts. When both paramesonephric ducts develop but the ducts fail to fuse at the 9th week of gestation, a duplicated system results [7]. The presence or absence of a vaginal septum is defined by the degree of fusion failure. According to the American Fertility Society Classification of Mullerian Anomalies, Uterus Didelphys is a class III anomaly [8,9].

Symptoms usually present shortly after menarche when hematocolpos develops during menstruation resulting in dysmenorrhea and a pelvic mass.

Understanding the imaging findings, in conjunction with the clinical presentation, is critical for early diagnosis in attempting to prevent complications such as endometriosis or adhesions from chronic infections with subsequent infertility. Management depends on the presence or absence of an obstruction. Surgical removal of the vaginal septum is the preferred approach for relieving an obstruction.

\section{References}

[1]. Carrington BM, Hricak H, Nuruddin RN, Secaf E, Laros RK Jr, Hill EC. Müllerian duct anomalies: MR imaging evaluation. Radiology 1990;176(3):715-720.

[2]. Pellerito JS, McCarthy SM, Doyle MB, Glickman MG, DeCherney AH. Diagnosis of uterine anomalies: relative accuracy of MR imaging, endovaginal sonography, and hysterosalpingography. Radiology 1992;183(3):795-800

[3]. Fielding JR. MR imaging of Müllerian anomalies: impact on therapy. AJR Am J Roentgenol 1996;167 (6):1491-1495.

[4]. Troiano RN, McCarthy SM. Müllerian duct anomalies: imaging and clinical issues. Radiology 2004; 233(1):19-34

[5]. O'Neill MJ, Yoder IC, Connolly SA, Mueller PR. Imaging evaluation and classification of developmental anomalies of the female reproductive system with an emphasis on MR imaging. AJR Am J Roentgenol 1999;173(2):407-416.

[6]. Li S, Qayyum A, Coakley FV, Hricak H. Association of renal agenesis and mullerian duct anomalies. J Comput Assist Tomogr 2000;24(6):829-834

[7]. Gray SW, Skandalakis JE, Broecker BH. Female reproductive system. In: Skandalakis JE, Gray SW, eds. Embryology for surgeons. 2nd ed. Baltimore, Md: Lippincott Williams \& Wilkins, 1994; 816-847.

[8]. Edmonds DK. Rokitansky syndrome and other Mullerian anomalies. In: Balen AH, Creighton SM, Davies MC, MacDougall J, Stanhope R, eds. Paediatric and adolescent gynaecology. Cambridge, England: Cambridge University Press, 2004; 267-274.

[9]. The American Fertility Society classifications of adnexal adhesions, distal tubal occlusion, tubal occlusion secondary to tubal ligation, tubal pregnancies, müllerian anomalies and intrauterine adhesions. Fertil Steril 1988;49(6):944-955. 